\title{
La perspective prélapsarienne de William Morris
}

William Morris's prelapsarian perspective

\section{Michel Remy}

\section{OpenEdition}

\section{Journals}

Édition électronique

URL : http://journals.openedition.org/rfcb/3304

DOI : $10.4000 /$ rfcb.3304

ISSN : 2429-4373

Éditeur

CRECIB - Centre de recherche et d'études en civilisation britannique

\section{Édition imprimée}

Date de publication : 1 septembre 2004

ISBN : 2-911580-19-2

ISSN : 0248-9015

\section{Référence électronique}

Michel Remy, "La perspective prélapsarienne de William Morris », Revue Française de Civilisation Britannique [En ligne], XIII-1 | 2004, mis en ligne le 01 septembre 2004, consulté le 07 octobre 2019. URL : http://journals.openedition.org/rfcb/3304 ; DOI : 10.4000/rfcb.3304

Ce document a été généré automatiquement le 7 octobre 2019.

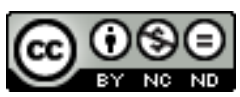

Revue française de civilisation britannique est mis à disposition selon les termes de la licence Creative Commons Attribution - Pas d'Utilisation Commerciale - Pas de Modification 4.0 International. 


\title{
La perspective prélapsarienne de William Morris
}

\author{
William Morris's prelapsarian perspective
}

Michel Remy

1 Comme de nombreux jeunes artistes des premières années du règne de Victoria, et en particulier les Pré-Raphaélites, William Morris succombe aux nouvelles idées esthétiques que Ruskin expose dans les volumes successifs du révolutionnaire Modern Painters (premier volume, 1843 ; cinquième volume, 1860), inaugurant littéralement une nouvelle perspective pour l'art ainsi qu'une redécouverte en même temps qu'une redéfinition des notions de beauté, de perfection et de vérité, notions débarrassées des artifices et dogmes guindés de la Royal Academy dont les références se résumaient encore à Michel-Ange, Raphaël et Poussin et sur laquelle planait toujours l'ombre de Sir Joshua Reynolds ${ }^{1}$. La réflexion suscitée par ces ouvrages, qui renvoient le lecteur à l'art $\mathrm{du}$ Moyen Âge et à l'art primitif italien, trouve d'inattendues confirmations et de singuliers échos dans diverses propositions théoriques de l'époque, souvent d'essence religieuse, comme si un consensus se formait quant à la nécessité d'un renouvellement profond des valeurs. Citons pêle-mêle l'oxford Movement, qui voit dans les rituels de l'Église catholique les gardiens de la vérité sacrée originelle et qui, en cherchant à introduire des éléments de la théologie romaine dans la religion anglicane, favorise un climat de controverses entre autres axées sur l'art; le Gothic Revival qui, rompant avec les théories habituelles de l'architecture, voit le retour au premier style gothique anglais comme le moyen de redonner à l'art anglais une authenticité nationale, autochtone; enfin, les ouvrages de Thomas Carlyle - dont Past and Present (1843), qui attaque les injustices sociales, économiques et politiques victoriennes à travers le portrait d'un abbé qui, grâce à son paternalisme charismatique, réforme un monastère en déréliction et lui redonne vie, recréant un univers à l'opposé du monde capitaliste industriel. De la même façon et avec autant de vigueur, les idées progressistes de Charles Kingsley tentent de réconcilier la science darwinienne et le dogme chrétien en un « socialisme chrétien » fondé sur la solidarité des travailleurs (Alton Locke, Tailor and 
Poet, 1850). Ces quelques éléments font partie des influences qui s'exercent sur le jeune étudiant d'Oxford qu'est Morris en 1853.

Ami intime de Burne-Jones qu'il avait rencontré sur les bancs de l'examen d'entrée à Exeter College en Juin 1852, il découvre avec lui la poésie d'exaltation morale de Tennyson et toute la richesse de la légende arthurienne dans la version de La Morte d'Arthur de Thomas Malory et Caxton, que Walter Scott avait popularisée à la fin du XVIII ${ }^{e}$ siècle siècle et dans laquelle les aventures des chevaliers ne sont plus éparses mais reçoivent unité narrative et cohérence thématique et morale. Ensemble, Morris et Burne-Jones voyagent sur le Continent, ensemble, ils décident de se consacrer à l'art et lorsque Burne-Jones commence à fréquenter l'atelier de Rossetti au début de 1854, il provoque naturellement la rencontre de Morris et de Rossetti ; ce dernier encourage Morris à peindre, qui, ainsi, abandonne sa brève carrière d'architecte.

3 La Confrérie pré-raphaélite, en tant que groupe, venait de se disloquer peu de temps avant, chaque artiste évoluant dans des directions plus ou moins différentes ${ }^{2}$. Mais l'impulsion donnée par Ruskin dès 1848 était toujours présente : les reconstitutions du passé, l'admiration pour Shakespeare, l'engouement pour le Moyen Âge, étaient devenus les principes fondamentaux d'un véritable manifeste pour l'exploration d'un passé où l'esthétique et l'éthique se rejoignaient, jonction qui hantera William Morris toute sa vie dans les domaines politique, social et artistique. Cette position est fortement corroborée lors de la publication de The Stones of Venice (vol. 1 en 1851 et vol. 2 en 1853), où, après avoir "exécuté » la Renaissance, Ruskin attaque l'industrialisation galopante et la division du travail qu'elle a entraînée. Le second volume de The Stones of Venice a particulièrement influencé Morris ${ }^{3}$.

\section{Les nouvelles valeurs, remémorées}

4 Ce qu'il faut retenir de cette plongée dans l'art médiéval, religieux surtout, c'est qu'elle s'accompagne d'une plongée dans les romans médiévaux de chevalerie, les ballades, les contes et légendes populaires et, en particulier, le cycle arthurien. En 1832, Tennyson publie ses Poems où figure 'The Lady of Shallott', histoire de l'amour de la Dame de Shallott pour Lancelot du Lac, amour non payé de retour. En 1842, Tennyson publie Sir Galahad, quête du Graal qui met en scène le fils de Lancelot et enfin, en 1859, Idylls of the King, qui fait d'Arthur l'incarnation symbolique de l'âme de l'homme idéal, déterminé à établir les vertus (victoriennes) de fidélité conjugale, de justice et d'action utile en faveur de la communauté, envers et contre les forces destructrices du matérialisme et du sensualisme. Lancelot est l'homme qui succombe à la tentation de la chair tandis que Guenièvre représente la sensibilité émotionnelle, naturelle, qu'il s'agit de maîtriser. L'ensemble de ces textes se veut annonciateur d'une nouvelle ère de vertu et de domination des passions. Mais, au-delà, ce qui sous-tend le succès de cette "reprise " du cycle arthurien, c'est bien le sentiment d'appartenance à une communauté qui partage les mêmes angoisses et les mêmes espoirs. À un moment où le lien social est violemment menacé par les problèmes socio-économiques et le développement de l'aliénation de l'homme par le travail, il ne pouvait en être autrement : le sentiment de fraternité dans l'effort et l'adversité, qui lie les hommes dans leur recherche de la vérité de la vie, se trouvait exalté dans l'exemple de la célèbre quête du Graal, au cours de laquelle le clivage entre le spirituel et le matériel s'efface progressivement à chaque épreuve dominée et s'abolit définitivement lorsque le Chevalier pur, Galahad, atteint 
son but et voit la Coupe sacrée du Christ Rédempteur. Cette nostalgie d'un état de choses rédimé qui repose sur la dépendance réciproque des hommes d'une même nation, est alors très forte, allant même se manifester dans les œuvres de Disraeli et le mouvement des jeunes conservateurs, Young England, dont le plaidoyer porte sur le retour à un comportement, dans le fond, féodal, où la solidité de la société repose entièrement sur des liens étroits entre les hommes d'une même communauté, identiques à ceux d'une famille, structure gardienne de sa propre spiritualité ${ }^{4}$.

5 Cet idéalisme, cette obsession d'une re-création pure, innocente, ce désir de réconciliation d'une communauté avec elle-même, vont nourrir toute l'œuvre de Morris et structurer toute son existence. Loin de tout escapism et de tout passéisme, Morris se lance dans la fabrication concrète d'objets et d'intérieurs qui tentent de créer un environnement propice à la réintroduction de ces nouvelles valeurs. Il s'agit là d'une remémoration qui se veut inscrite dans le devenir, une sorte de remémoration de ce qui n'est pas encore, si l'on veut. Ce sera l'esprit dans lequel ses romans utopiques, The Dream of John Ball et News from Nowhere seront écrits.

\section{Iseult ou Guenièvre?}

6 Ces nouvelles valeurs, on les trouve en filigrane - nous pensons à l'insu de leur auteur dans le seul tableau pré-raphaélite de Morris, d'abord intitulé La Belle Iseult, puis Queen Guinevere (1858). Qu'importe les raisons personnelles de cette confusion. Pour nous, c'est le passage du sens du premier titre au sens du second qui peut nous renseigner sur le travail profond de Morris. C'est en effet le passage du thème de la frustration amoureuse et de l'attente du bien-aimé au thème de la séduction et de la trahison qui introduit le chaos dans le royaume. Guenièvre est dans sa chambre où règne un singulier désordre, ainsi que l'indique le lit défait aux draps repoussés, image de son propre état mental. Elle n'a pas fini de s'habiller et doit encore boucler la ceinture qui maintiendra sa robe. Ironique symbole de fidélité, le chien occupe maintenant le lit que, vraisemblablement, Lancelot vient de quitter. L'expression de confusion mentale, sinon d'angoisse sur son visage, trahit sa prise de conscience des conséquences de son acte : cet amour illégitime sera la cause de la désagrégation des liens organiques tissés entre le Roi et ses chevaliers et la perte des valeurs qui cimentaient l'unité de la communauté. Cette obsession de l'unité est telle qu'elle pousse Morris à tenter par ailleurs de disculper la reine de son acte et de la justifier, dans son poème The Defence of Guinevere, au nom de la nature créée par Dieu et de l'innocence fondamentale de la passion vraie et spontanée. Guenièvre est une nouvelle Ève dont il importe pour Morris de relativiser, dirions-nous, la responsabilité dans la rupture de l'ordre social'

7 Mais regardons le tableau de plus près. Certes, le traitement de Guenièvre est l'expression même, majestueuse, du style cher à Rossetti et aux Pré-Raphaélites, qui redonne à la femme une présence forte, imposante, tout en étant mystérieuse, l'expression d'une femme complexe, présente et lointaine, sensuelle et abstraite, promesse de passion et de froide résistance, dépositaire des secrets de l'âme et du corps ; certes, le pré-raphaélitisme se retrouve dans la richesse et la chaleur des tons, le nombre des détails, les fruits, le missel enluminé et le pot à eau qui revient dans d'autres tableaux, signe de possible purification. Mais ce qui frappe, c'est le souci précis, non pas de la nature symbolique des objets disséminés ici ou là, mais de leur dessin, de leur texture, de leurs motifs. Ils forment tous une toile de fond, un véritable 
hypotexte, sur lequel se découpe Guenièvre, comme un palimpseste dont le texte caché reviendrait à la surface. Tout ce qui se répète, le motif de l'arbre chargé de fruits (allusion à l'arbre de vie au centre du Jardin d'Éden), le motif floral de l'étoffe qui recouvre la table de toilette, le motif du tapis et du couvre-lit, tous constituent les constantes d'un tableau dont le sujet est l'inconstance, les certitudes d'un univers d'incertitudes et de duplicité. Le couvre-lit est «menacé » par le désordre des draps du lit. Ce sont, comme le drapeau fixé au mur, les évocations d'un monde de valeurs avec lequel il faut désormais renouer, lorsque Gueniève aura renoué sa ceinture...

Ce tableau est donc à lire au moins doublement. D'abord dans son appartenance à une symbolique intrinsèque à la Confrérie pré-raphaélite et à sa quête de valeurs rédemptrices, fidélité, solidarité, pureté; ensuite, dans son évocation des autres intérêts de Morris et de son travail de traduction de ses idées dans le domaine des arts appliqués, décoration intérieure, mobilier, tapisseries murales, tissus, tapis...aucun de ces arts n'ayant plus d'importance l'un que l'autre, tous ces arts constituant les éléments fondateurs d'une sorte de contrat social et spirituel.

\section{Vers une communauté idéale, remémoration de l'à- venir}

On connaît l'influence de l'enseignement de Ruskin sur William Morris et son insistance sur la fidélité à la nature, sur le respect du principe vital de celle-ci et sur le devoir qui incombe à l'artiste de rendre compte de ce principe afin que son public retrouve les valeurs primordiales dont la corruption de la société l'éloigne. C'est dans ce sens que Ruskin ne cessait de préconiser l'alliance étroite entre les différents arts et l'environnement immédiat de l'homme, entre son activité et sa sensibilité artistiques et son activité artisanale et quotidienne, concrète, et de même que l'architecte devait prendre en compte l' " esprit du lieu », de même il devait maîtriser et coordonner les différents corps de métier: peintres, ferronniers, carreleurs ou vitriers. Cette image d'une collaboration harmonieuse, cette unité à travers la diversité, pour peu que les règles soient justes et acceptées par tous, inspire tout le travail de Morris.

En 1859, Morris fait construire une maison à Upton, près de Bexleyheath, dans le Kent, qu'il appelle Red House, de la couleur des briques utilisées, résultat d'un travail parfaitement collectif. Philip Webb, ami de longue date, en est l'architecte, Rossetti décore l'intérieur et fait même quelques meubles, dont des chaises qui combinent une forme empruntée aux chaises de ferme et un matériau précieux, l'ébène, le cannage, fait à la main, étant en roseau naturel. Burne-Jones fait d'autres sièges, inspirés de motifs médiévaux, Morris dessine et fabrique les tentures brodées aux motifs d'oiseaux, de fleurs et d'arbres dessinés par Philip Webb. Dans le jardin, dessiné par Morris, vergers et parterres sont séparés par des plates-bandes d'herbe et des espaliers de rosiers en imitation de l'époque élisabéthaine. Enfin, couronnant cette activité collective, Rossetti est invité, après la mort d'Elizabeth Siddall en 1862, à y séjourner avec Morris et Jane, qui savait le réconforter...

11 Ce partage, ou plutôt cette fusion des tâches évolue très rapidement en projet commercial d'une entreprise collective, que Morris voulait modèle du genre. Épouses et compagnes y participent. En 1861, il fonde la firme Morris, Marshall, Faulkner and Co, 
dont le but, inscrit sur les prospectus, est de créer 'harmony between the various parts of a successful work'. Du point de vue que nous adoptons ici, le mot harmony est crucial.

La suite est une série d'initiatives de Morris pour consolider et développer ces nouvelles conditions de vie et de travail. En 1865, la firme déménage de Red Lion Square pour s'établir dans Queen Square, dans le quartier de Bloomsbury, les commandes publiques commençant à affluer, parmi lesquelles la restauration de la salle des armes et des tapisseries de St James' Palace et la décoration intérieure de la salle à manger du Musée de South Kensington. En 1871, Morris acquiert une résidence près de Lechlade dans l'Oxfordshire, Kelmscott Manor, lieu qui correspond parfaitement à son idéal de vie rurale paradisiaque. Il y installe son atelier, demande à Rossetti d'être son colocataire et transforme plusieurs pièces en ateliers de tapisserie, de tissage de tapis et de carpettes nouées à la main.

En 1873, dans la maison de Queen Square, il installe une petite teinturerie après s'être lancé dans une série d'expérimentations en matière de teinture et d'impression de tissus. En 1874, à la suite d'une restructuration de la firme, il décide de la dissoudre, rachète les parts de ses partenaires et assure ainsi seul le contrôle de toutes les opérations et activités, rebaptisant la firme Morris and Co.

En 1878, il déménage avec sa famille dans Hammersmith, dans une grande maison qu'il rebaptise Kelmscott House, comme s'il voulait signifier la parfaite continuité de l'expérience collective, l'absence de toute rupture et la confirmation de l'identité et de l'intégrité de l'expérience. Cette maison et ses dépendances deviennent une véritable ruche d'activités de plus en plus diversifiées, mais au projet toujours unique, tant et si bien qu'il lui faut louer un autre lieu à Merton Abbey, à dix kilomètres de Londres, afin de pouvoir embaucher lui-même de nouveaux ouvriers spécialisés et de se soustraire à la dépendance vis à vis de sous-traitants. Il travaille en particulier avec William de Morgan, céramiste réputé. Seul maitre à bord, auteur in fine de tout ce que produit l'entreprise, Morris devient un auteur collectif, pluriel, garant de l'harmonie et de l'unité, sorte de démiurge des arts appliqués.

\section{L'individu et le travail}

15 Tout au long de cette expansion et de toutes ces expériences, Morris ne cesse de penser à la condition ouvrière et aux problèmes sociaux qu'implique le travail. Comment son idéal de collectivité, de travail communautaire, pouvait-il se traduire en termes concrets ? Comment se caractérisait le lieu de ce travail ? En 1872, lorsqu'il commence à s'intéresser à la teinturerie, il se déplace à Leek, dans le Staffordshire, chez Thomas Wardle qui l'initie à cette nouvelle activité et il peut constater les effets de ce pénible labeur chez les ouvriers qui travaillent sur la chaîne de production. Convaincu alors de l'importance humaine de ne pas séparer les tâches, reprenant les idées de Ruskin, à la division du travail, il déclare préférer l'implication des ouvriers dans les différentes tâches, faisant de ceux-ci des participants à part entière, collaborateurs l'un de l'autre, plutôt que des exécutants limités à une seule tâche. L'esprit de collaboration et de communauté ne quitte Morris à aucun moment.

16 C'est à Merton Abbey qu'en 1881 il s'approche le plus de cet idéal de phalanstère, si l'on peut emprunter ce terme à Fourier en l'édulcorant quelque peu. Y installant les différents corps de métier dont il dépend - tisserands, tapissiers, céramistes et verriers - il instaure une organisation précise du travail qui se soucie avant tout du bien-être 
des ouvriers. Les employés sont correctement payés, travaillent moins que dans de nombreuses autres entreprises, disposent d'une bibliothèque itinérante, d'un dortoir et une formation professionnelle est réservée à chacun des jeunes employés. Les enfants de 13 ou 14 ans à qui est confié le tissage des tapisseries sur des métiers de haute lisse reçoivent le gîte et le couvert, ainsi qu'un salaire hebdomadaire. Un système de participation aux bénéfices est mis en place pour les plus anciens des employés qui occupent différents niveaux de la hiérarchie, ce qui n'est pas sans rappeler les propositions sociales de Ruskin concernant les travailleurs, leur bien-être, leur retraite et leur cadre de vie. Les champs et les jardins sont expressément plantés d'arbres fruitiers et de légumes qui servent à la communauté. Rêve d'autarcie ou utopie socialiste?

Nous arrivons ici sur le terrain de l'engagement politique, qui n'entre pas totalement dans notre propos, mais qui servira à corroborer nos premières observations. D'abord, à l'enthousiasme militant du début, succède une période de confusion qui finit, Morris avançant en âge, par une lassitude devant la fragmentation du mouvement socialiste en plusieurs factions. Puis, après avoir lu Le Capital de Marx en 1882, qui le convainc de mener une action fortement revendicative qui n'exclue pas le recours à des actions illégales, Morris devient membre de la Democratic Federation (la fille de Marx, Eleanor, en était membre). Le changement radical, c'est-à-dire la suppression de toute hiérarchie, prôné par la Fédération amène Morris à s'interroger sur l'équilibre à établir entre liberté individuelle et pouvoir étatique. Commence alors une période où il donne conférence sur conférence, exposant sa vision socialiste de l'avenir, fondant ses arguments sur la nécessité du partage, de la suppression des classes sociales et de l'instauration d'une société égalitaire et démocratique, comme, pense-t-il, aux premiers temps de l'humanité. Passant de radical à révolutionnaire, il se sépare de la Democratic Federation pour former en 1884 la Socialist League avec plusieurs autres dissidents, dont Eleanor Marx. La Ligue, divisée en 19 sections, était en fait pilotée par la dix-neuvième, celle de Hammersmith, dirigée par Morris lui-même et dont le siège était à Kelmscott House. Morris écrit alors beaucoup pour son organe de presse, Commonweal, tient de nombreuses réunions mais laisse la Section être infiltrée par plusieurs anarchistes, dont le célèbre prince Piotr Alexéievitch Kropotkine et le nihiliste Sergius Stepniak, cédant ainsi à son penchant naturel du refus de toute autorité sinon collective. Mais ses idées prévalent de moins en moins et il est renvoyé de Commonweal après la publication, dans ses pages, du dernier épisode de News from Nowhere en 1890. Morris n'en arrête pas pour autant discours, conférences et autres prises de parole. Jamais il n'abandonna son idéal de communauté des hommes, voyant d'une part dans le socialisme d'État une phase, une étape, et d'autre part dans sa propre expérience le modèle sur lequel fonder un tel idéal.

\section{Le Paradis perdu, mémoire profonde du futur}

Reprenons tout ce qui précède afin d'essayer de saisir la vision profonde de William Morris, ce non-dit qui sous-tend toutes ses expériences et expérimentations. Certes, il est aisé d'y lire le désir communiste d'une société sans classe et d'une égalité de tous devant le travail et ses profits, nous l'avons amplement dit, mais il ne faut pas oublier une autre dimension, non plus politique et sociale, mais spirituelle et symbolique. Car il y a un nœud que Morris a essayé de dénouer, une image, toujours fuyante qu'il a tenté 
d'arrêter et de traduire en faits, c'est l'image obsédante du Jardin d'Éden, le jardin de l'homme et de la femme avant la chute. Commençons par les deux dimensions de son œuvre, l'espace et le temps, car c'est de cela qu'il s'agit: redonner à l'homme un espace-temps où il soit respecté en tant que sujet, au sens philosophique du terme. Dans quel espace Morris évolue-t-il ? Dans quelle temporalité séjourne-t-il ?

Il est clair que l'espace dans lequel le travail de Morris s'effectue est un espace constitutivement clos, même s'il apparait en expansion. Que ce soit dans la décoration intérieure fondée sur tapis, papiers peints ou tapisseries, y compris papiers peints spéciaux pour plafonds, ou même dans sa calligraphie et l'utilisation de larges bordures lourdement décorées qui enserrent et encadrent les textes des éditions de la Kelmscott Press, l'espace est obsessionnellement cadré, clôturé. À meilleure preuve, l'étonnante première page de News from Nowhere, où la perspective est ramenée vers l'avant par le texte et surtout par les volutes qui l'entourent. Le cadre, par sa forte présence, sa violence, nous fait prendre conscience de l'espace unique, isolé, sacré qu'il encadre, un jardin bien tenu ; il le sépare de notre monde tout en en faisant partie par le motif de sa décoration étranger à la scène qui y est représentée. Le cadre est un seuil sacré, il fonctionne comme une iconostase, signalant qu'il donne accès à la profondeur, ici spirituelle, de la réalité, tout en l'isolant de l'extérieur, de l'hétérogène, de ce qui pourrait en menacer l'intégrité.

L'espace morrissien tend donc à la clôture et à la planéité. Certes, on retrouve ici le trait caractéristique de l'esthétique pré-raphaélite qui, en écrasant les perspectives, surtout chez Rossetti, veut précipiter l'œuvre et l'œil du spectateur l'un dans l'autre en refusant à ce dernier toute échappée vers le «fond». Mais cela devient encore plus évident chez Morris où les scènes historiques et médiévales ou les évocations de la nature de ses tapis, tapisseries et papiers peints apparaissent comme particulièrement aplaties, dénuées de tout relief et de toute profondeur. L'enchevêtrement des branchages, la profusion des fleurs et des feuillages, la couleur toujours assez sombre des fonds créent un espace plat, totalement bidimensionnel, qui, bien sûr, nous renvoie à nous-mêmes, utilisant toute la lourde matérialité des murs pour nous y enclore. Le rythme que créent ces motifs s'étire et s'étale à la surface des murs ou des planchers, linéairement, nous maintenant en leur intérieur.

21 Or pointe ici une intéressante ambiguïté. Car le leitmotif des dessins de Morris et de ses collaborateurs, c'est la nature, la vie, le développement des formes. Pas un motif de ses broderies, tapisseries, carrelages ou papiers peints qui ne soit un arbre en pleine santé, des fleurs épanouies d'une infinie variété, fleurs de fraisiers, roses, chardons, tulipes, chèvre-feuille, tagètes, narcisses, églantines, fleurs d'artichaut, lis, coquelicots, jasmin, la liste est sans fin, véritable tentative d'exhaustivité dans le recensement de la création divine. Or cette abondance florale, cette prolifération des formes, hymne vibrant à la croissance organique et à la vie, semble bien s'opposer à l'impression de clôture et de claustration que donne la planéité du dessin et qui devait créer dans la pièce ou le salon un espace fermé sur lui-même. C'est là le paradoxe de l'espace de Morris où n'existent ni haut ni bas, ni droite ni gauche. Espace en pleine répétition de lui-même, qui renvoie sans cesse au geste qui l'a créé, geste d'application mille fois répété du même tampon sur la toile tendue ou le papier. Les fleurs nous renvoient aux fleurs, les murs aux murs, la vie à la vie, en une circularité spéculaire. Rappelons à ce propos que Morris critiquait violemment l'habitude victorienne qui consistait à juxtaposer sur un mur des lais de papiers peints de motifs différents. L'espace de Morris 
est donc l'espace d'un jardin secret, d'un hortus conclusus, espace de célébration de l'organicité et de la croissance, mais d'une croissance qui ne déborde pas, comme si ses valeurs, sa vérité, en pourraient être d'autant menacées ${ }^{6}$.

Il est dès lors évident que la temporalité de cet espace est une temporalité de l'entredeux, où l'écoulement du temps est arrêté, une sorte de temps a-historique, un temps qui échapperait à l'histoire. D'abord, les scènes qui sont évoquées sont celles d'un temps "mythologisé ", d'une époque médiévale arrachée à son temps historique et transformée en symbole, en valeur. Lorsque Rossetti conçoit les chaises pour Red Lion Square, non seulement le modèle est médiéval, mais elles sont recouvertes de scènes peintes inspirées du poème de Morris sur Galahad, glorifiant un espace bucolique, une Arcadie habitée par des chevaliers nobles et des dames vertueuses. La temporalité est celle, spirituelle, mythique, symbolique, du Graal, éternellement recherché.

Ensuite, comme nous venons de le dire, la répétition des motifs, la répétition du Même, écrase le temps chronologique en un présent sans devenir, un présent qui n'aurait plus de passé ou de futur. Il est curieux que lorsque Morris décide de se lancer dans l'imprimerie afin de publier des ouvrages d'art et pour bibliophiles en 1888, il se rend à la Bibliothèque bodléienne à Oxford et entreprend de créer deux nouveaux caractères d'imprimerie, le Golden, inspiré d'un modèle romain vénitien du XV siècle, et le Troy, inspiré des caractères gothiques, auquel on peut ajouter le Chaucer, sa version réduite : tentatives de continuation du passé dans le présent, de re-création du présent d'alors dans un présent éternel. Temporalité du comme si, comme si c'était hier, comme si tout recommençait, comme si nous étions maîtres du temps, comme si seule importait la croissance organique, pure, sans naissance ni mort, temporalité ancrée dans l'atemporalité, comme à la naissance du monde si l'on en croit la Genèse.

\section{Conclusion}

C'est cet espace et ce temps primordiaux, pour ainsi dire, que Morris tente de recréer dans les locaux où il travaille, en installant la vie coopérative, communautaire que nous avons décrite, prenant modèle très certainement sur les corporations du Moyen Âge, vision-cathédrale s'il en est, où ce qui importe, n'est pas ce qui est fait, mais le faire, non pas le fini, mais ce qui le précède, l'exaltation du construire. À chacune de ses expérimentations, Morris ne privilégie jamais le moment où l'œuvre sera réalisée et finie. Son principal souci, c'est le savoir-faire du faire. Refusant les techniques modernes de teinturerie à l'aniline, par exemple, il se met à rechercher de vieilles recettes de teintures naturelles pour ses tissus imprimés, consulte des ouvrages anciens de la Renaissance et passe des mois entiers à essayer différentes combinaisons. En 1877, il refuse les méthodes mécanisées de production commerciale de tapisseries qui le limitent trop dans sa créativité foisonnante et il revient à des méthodes traditionnelles de tissage de haute lisse. En 1857, pour l'activité de broderie de son entreprise, il refuse l'utilisation des fils de soie que préconisait son ami Street et préfère les fils de laine, plus difficiles à travailler, mais qui se rapprochent davantage des broderies médiévales. Après avoir passé énormément de temps à maîtriser cette technique, il brode lui-même puis devant le nombre de commandes, il instruit sa gouvernante ainsi que Jane, Georgiana Burne-Jones, Lucy et Kate Faulkner, parfaite fusion de la théorie et de la pratique. C'est le temps mis à réaliser, le temps avant la réalisation, ce temps intermédiaire, qui est porteur et expression des valeurs qui assurent la dignité de 
l'homme, valeurs retrouvées, récompense réelle, à la fois matérielle et spirituelle du travail fourni. Adam, heureux ouvrier du Créateur dans un paradis qui ne serait plus perdu.

Dans cet espace clairement délimité, le temps est suspendu. Rêve de totalité, espace clos de croissance, espace fini entre des murs sur lesquels s'étale l'infini, temporalité qui bégaie, le lieu du travail de Morris est un lieu qui multiplie ses topoi, un lieu nonlieu, un u-topos, à la fois harmonieux (eu-topos ?) et pas-encore-là, dont la prolifération végétale et animale ne peut que nous renvoyer au lieu primordial du Jardin d'Éden, quand le temps n'était pas encore, jardin paradisiaque, comme le jardin-seuil de la première page de News from Nowhere ramené à nous pour que l'on séjourne dans cette perspective arrêtée et murée par le lourd encadrement, lieu offert, monde d'harmonie des corps et des âmes, en une remémoration permanente de l'image du Paradis de Milton, monde qui n'est nulle part mais qui peut être partout re-créé, lieu d'une création perpétuelle qui se répète elle-même, sans heurt ni rupture, monde du Earthly Paradise ${ }^{7}$. La vision de Morris, telle qu'elle s'est exprimée dans son travail de conception de l'entreprise, dans son action sociale et politique et dans son esthétique, est, on l'aura compris, fondamentalement prélapsarienne. C'est là, à notre sens, la clé de toute son œuvre.

\section{Appendice : Note sur The Earthly Paradise et l'utopie}

Refusant toute moralisation stricte, ce long poème, composé à partir de 1865 et publié avec un énorme succès en 1868, peut être considéré comme une épopée pré-raphaélite qui cristallise le désir régressif d'un monde pur et parfait. Si le thème est clairement la désillusion que l'on rencontre inévitablement dans toute aspiration à la célébrité, à la richesse et à la sensualité, le texte vaut surtout pour sa traversée du temps et de l'espace qu'assure la narration des différentes histoires, les unes se référant à la mythologie grecque, les autres à la mythologie scandinave, telle autre à des légendes arabes; il vaut aussi pour la façon dont s'établit, à la faveur de cette traversée, un ensemble de valeurs universelles, atemporelles, qui appartiennent autant à telle partie du monde qu'à telle autre, autant au passé qu'au présent. Il s'agit là d'une remontée vers des lieux et des temps de convivialité, de fraternité et d'hospitalité de l'autre, 'where tossed about, all hearts of men must be', tout ceci participant parfaitement du processus de remémoration dont nous avons parlé. L'introduction elle-même (qui suit l'Apologie) demande au lecteur d'oublier "la fumée ", "la vapeur", le bruit des pistons, l'urbanisation tentaculaire, et lui assigne cette attitude :

Think rather of the pack-horse on the down

And dream of London, small and white and clean,

The clear Thames bordered by its gardens green,

Think, that below bridge the green lapping waves

Smite some few keels that bear Levantine staves. (v. 4-8)

C'est là, dans cet ailleurs, que Morris dit vouloir «installer ses rimes " et créer un monde de beauté, d'amour et de paix, où la vie et la mort se relient en une boucle sans fin : 'Fair death of things that, living once, were fair'.

Cette aspiration quasi sotériologique est centrale à la problématique de l'utopie qui, chez Morris comme chez d'autres utopistes, trouve son origine dans un certain type de discours, c'est-à-dire un système de pensée, qui convoque le réel, le ramène à lui, le 
réduit même, afin que le discours en question, le système ou le modèle proposé, soit légitimé, accepté, reconnu, institutionnalisé. La boucle est bouclée, le système utopique se clôt sur lui-même et fonctionne pratiquement en autarcie.

L'œuvre polymorphe de Morris fonctionne ainsi sur une sorte d'antéposition du discours, une description d'un certain monde qu'il s'est ensuite employé à rendre réel dans tous ses aspects économiques, sociaux, humains, politiques, éthiques même. La jubilation de Morris, son industrie, au sens étymologique, son inventivité proliférante, son enthousiasme si étonnamment créatif naissent de cette anticipation du réel, de ce désir de le faire sien, transformant sa nostalgie d'un bienheureux état primordial en la croyance ferme et définitive dans son propre pouvoir d'instaurer cet état envers et contre toute autre autorité. On peut se demander si, ici, Morris ne se situe pas à la limite hautement problématique, sinon dangereuse, du narcissique et du totalitaire, qui, de toute façon, nous semble-t-il, court le long de tout texte utopique et le sous-tend. Après tout, le paradis terrestre n'est-il pas censé être le nombril du monde?

\section{NOTES}

1. Le titre complet du livre de Ruskin représente bien une rupture: Modern Painters: Their Superiority in the Art of Landscape Painting to All the Ancient Masters Proved by Modern Artists, Especially J.W.Turner. Le rejet des « anciens maîtres » constitue l'exact contrepied des principes de Reynolds.

2. Rappelons que la première réunion de la Confrérie pré-raphaélite s'était tenue dans l'atelier de Millais en Septembre 1848 avec Hunt, Rossetti, Millais et quatre autres artistes, William Michael Rossetti, James Collinson, Thomas Woolner et F.G. Stephens.

3. Dans le deuxième volume, Ruskin fait l'éloge du travail de création, dénonce l'aliénation du travailleur par la machine qui lui enlève toute réflexion et tout rapport personnel avec ce qu'il produit, et donc ne crée plus. Cette idée sera centrale à toute la démarche de William Morris.

4. Voir, en particulier, la «trilogie» de Benjamin Disraëli : Coningsby (1844), Sybil, or The Two Nations (1845) et Tancred (1847).

5. Il est intéressant que, dans son plaidoyer pro domo, Guenièvre, devant ses juges, non seulement se réfère à Dieu comme étant au fond l'inspirateur de ses actes mais précise que sa passion pour Lancelot a éclaté au moment d'une sorte de fusion spirituelle avec la Nature. (The Defence of Guinevere, v. 109-141). Plus troublant est le rapport de ce tableau à la réalité du couple que forme alors William Morris et Jane Burden. On sait que Jane Burden, le modèle d'Iseult-Guenièvre, avait été rencontrée par hasard par Rossetti et Burne-Jones dans un théâtre d'Oxford. Rossetti lui demanda de poser pour eux, et elle devint bientôt le prototype de la beauté pré-raphaélite, fusionnant ainsi la réalité et l'art. Morris tomba éperdument amoureux de Jane Burden, mais celle-ci aimait Rossetti qui ne pouvait lui retourner son amour, étant lié à Elizabeth Siddall, que sa maladie retenait à ses côtés. Jane épousa Morris en 1859 un peu par dépit et déception, mais à la mort d'Elizabeth Siddall, un étrange ménage à trois se constitua, abolissant en quelque sorte la contradiction que dressent les deux titres du tableau en mêlant Iseult et Guenièvre en une seule figure unitaire. Plus tard, Morris devint le défenseur de l'amour libre, notion qui vise à abolir également les contradictions. 
6. Autre confirmation de ce leitmotif de la clôture, de l'espace homogène qui veut sans cesse acclimater l'hétérogène, le lieu où Guenièvre découvre la beauté de la Nature : 'I was half mad with beauty on that day,/ And went without my ladies all alone,/ In a quiet garden walled round every way;/ I was right joyful of that wall of stone,/ That shut the flowers and trees up with the sky,/ And trebled all the beauty ...[v. 109-114]' (nous soulignons). La beauté inséparable d'un monde harmonieux car clos, ou même clos car harmonieux, n'est pas sans rappeler, quasi littéralement, la description du Paradis par Milton dans le Livre IV de Paradise Lost.

7. The Earthly Paradise (1868) consiste en 24 longs poèmes narratifs, deux pour chaque mois de l'année, totalisant 42000 vers et mettant en scène des voyageurs vikings, survivants d'une épidémie de peste, qui cherchent un paradis terrestre et rencontrent sur une île les descendants d'un groupe de Grecs qui s'y étaient installés longtemps auparavant. La scène est censée se passer au XIV ${ }^{e}$ siècle, les voyageurs et leurs hôtes se racontant alternativement des histoires du passé (voir Appendice).

8. Selon le vers 40 de l'Apologie.

\section{RÉSUMÉS}

La vision de Morris, telle qu'elle s'est exprimée dans son travail, dans sa conception de l'entreprise, dans son action sociale et politique et dans son esthétique totalisante, tend à l'établissement d'un univers qui, en voulant sans cesse arraisonner l'hétérogène, se clôt sur luimême afin de préserver son unité et son harmonie. La «figure » qui se loge au creux du texte morrissien, sa perspective si l'on veut, semble bien être celle d'un Paradis qui ne serait plus perdu, entraînant ainsi le regardeur et le lecteur dans une perspective prélapsarienne, clé de toute son œuvre.

Morris's vision, as expressed in its conception of collaborative work, in its social and political activities and in its all-comprehending aesthetics, creates a universe which, although expanding, repeatedly closes upon itself in order to safeguard its unity and harmony. Morris's perspective is that of a paradise which would not be lost any longer, a prelapsarian perspective, the key to all his work.

\section{AUTEUR}

\section{MICHEL REMY}

Université de Nice-Sophia Antipolis 